\title{
A rare cause of coffee-ground vomiting: Retrograde jejunogastric intussusception
}

\section{Kiran Gangadhar, $M D$}

Department of Radiodiagnosis and Imaging, Institute of Medical Sciences, Banaras Hindu University, Varanasi, India

Corresponding author: K Gangadhar (kirang.585@googlemail.com)

\begin{abstract}
Retrograde jejunogastric intussusception is a well-recognised, rare, but potentially fatal long-term complication of gastrojejunostomy or Billroth II reconstruction. Only about 200 cases have been reported in the literature to date. Diagnosis of this condition is difficult in most cases. To avoid mortality, earlydiagnosis and prompt surgical intervention is mandatory. Since gastrojejunostomies with vagotomy are on a declining trend, it is extremely rare to come across such a complication. We report on such a patient who presented with haematemesis.
\end{abstract}

S Afr J Rad 2012;16(2):72-73

\section{Case history}

A 60-year-old man was admitted, complaining of abdominal pain after 4 days of coffee-ground vomiting. He had undergone gastric surgery for peptic ulcer disease 25 years earlier, but details of his surgery were not available at the time of admission. The patient underwent a gastrograffin swallow and computed tomographic (CT) examination for further evaluation. We considered whether the diagnosis might have been possible by the gastrograffin examination alone.

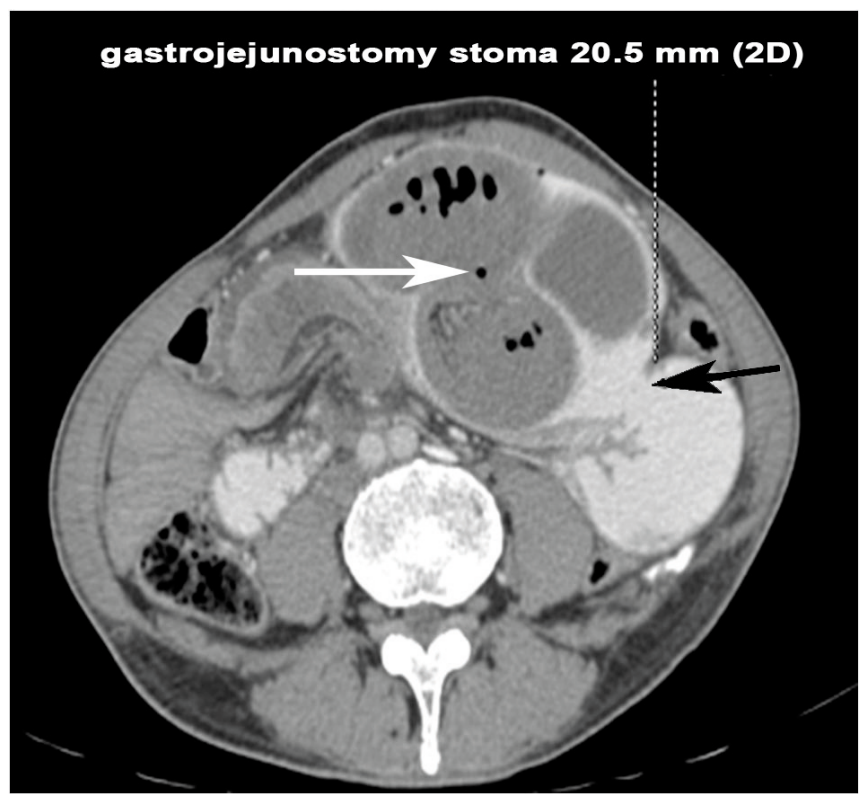

Fig. 1. Axial CECT of the patient showing retrograde intussusception of the jejunal loops within the stomach, the black arrow pointing towards the gastrojejunostomy stoma and its size shown by the broken line. A small intramural air density was noted in the intussuscepted bowel (white arrow). Efferent loop of the gastrojejunostomy site involved is diagnostic of type II variety.

\section{Discussion}

Retrograde jejunogastric intussusception (JGI) is a rare but serious complication following gastric surgery, where small bowel loops become incarcerated and strangulated inside the stomach. ${ }^{1,2}$ The condition was first described by Bozzi in a patient who had undergone gastroenterostomy. ${ }^{3}$ Eight years later, the complication was also reported in a patient with Billroth II resection. Subsequently, around 200 isolated cases and small series have been published. ${ }^{1-4}$ JGI seems therefore to be a rare complication after gastrojejunostomy or Billroth II gastrectomy. It also has been described as a complication following previously placed gastrostomy tube, total gastrectomy, Billroth I operation ${ }^{1}$ and Rouxen-Y gastric bypass. The widely accepted anatomical classification proposed by Shackman ${ }^{5}$ distinguishes 3 categories of JGI: Type I - afferent loop intussusception (antegrade); Type II - efferent loop intussusception (retrograde); and Type III - combined form. Type II, or retrograde efferent loop intussusception (Fig. 1), is the most common (80\%), with the 2 other types accounting for $10 \% .{ }^{1}$ In the case presented here, the type II JGI was documented.

The causes of JGI are poorly understood. Various factors have been implicated, such as hyperacidity, long afferent loop, jejunal spasm with

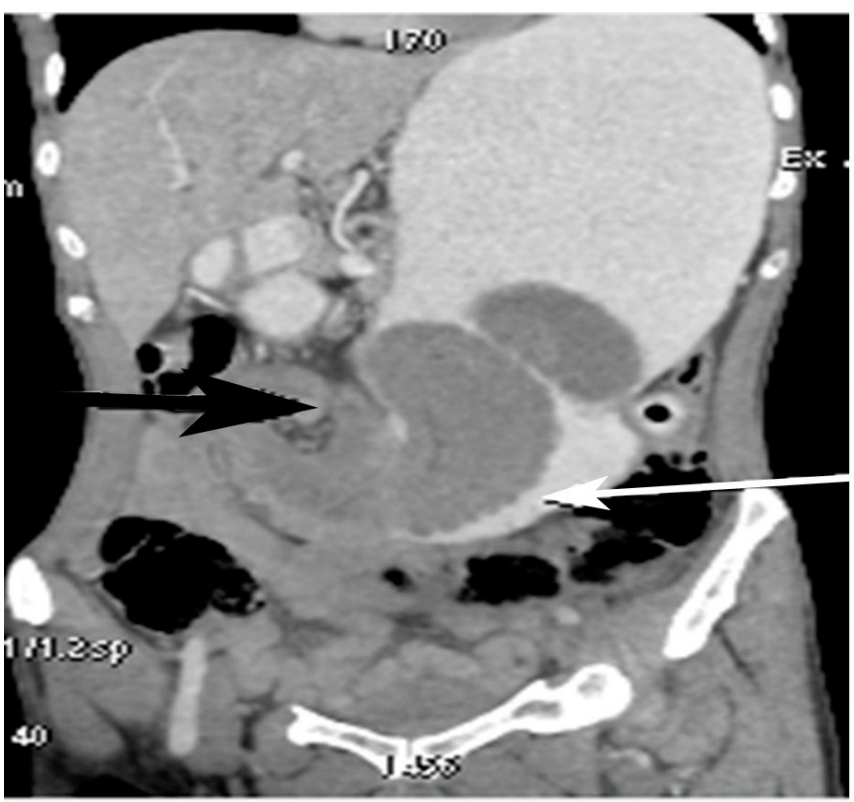

Fig. 2. Coronal CECT of the patient showing retrograde intussusception of the jejunal efferent loops (white arrow) within the stomach, and a central hypodense (black arrow) area is seen giving a fat density, suggestive of mesenteric fat with mesenteric vessels that had intussuscepted along with the loops. 
abnormal motility, increased intra-abdominal pressure, retrograde peristalsis, etc. Retrograde peristalsis, which can occur in normal people prior to gastric surgery, seems to be accepted as the cause of type II JGI. If not suspected, the clinical picture can be quite non-specific and the possibility of intussusception may not even be considered. The dominant symptom is pain, occasionally associated with nausea and vomiting. Patients may present with high intestinal obstruction or severe haematemesis from secondary ulceration. ${ }^{6} \mathrm{~A}$ firm mass may be palpable in the epigastrium. A water-soluble upper GI contrast study may reveal a 'coiled spring' appearance within the stomach. Upper GI endoscopic examination is often diagnostic and may visualise the jejunal segments as they migrate in and out of the stomach. When a patient presents with haematemesis and has a mobile upper abdominal mass with visible peristalsis, and bears an upper midline or paramedian scar, one should suspect this complication first. ${ }^{6}$

The treatment for JGI is surgical intervention as soon as possible. Surgical options include reduction, resection and revision of the anastomosis, depending on the conditions found during the operation. The best way to prevent recurrence, if any, has not been identified yet.

\section{Conclusion}

Retrograde JGI is a very serious life-threatening complication of gastric surgery. There is a wide variation in the lapse time between the gastric surgery and occurrence of JGI, as seen in the present case. When a patient who has had history of gastric operation presents with epigastric pain, vomiting and haematemesis, the possibility of JGI should be considered along with more common diagnoses such as a recurrent stomach ulcer. Because this condition is life-threatening, awareness of this rare complication is essential to save lives by operational intervention.

1. Archimandritis AJ, Hatzopoulos N, Hatzinikolaou P, et al. Jejunogastric intussusception presented with haematemesis: a case presentation and review of literature. BMC Gastroenterol 2001;1:1-4.

2. Bapaye M, Kolte S, Pai K, et al. Jejunogastric intussusception presenting with outlet obstruction. Indian [ Gastroenterol 2008;22:31-32.

3. Bozzi E. Annotation. Bull Acad Med 1914;122:3-4.

4. Hasan M, Mahamud MM, Khan SA, Rahman M. Jejunogastric intussusception. Mymensingh Med J 2009;18(2):255-259.

5. Shackman R. Jejunogastric intussusception. Br J Surg 1940;27:475.

6. Menezes LT, D'Cruz A. Retrograde jejunogastric intussusception following gastric surgery. J Indian Med Assoc 1986;84:310-311. 